\title{
Recurrent episodes of ventricular tachycardia in the patient with LV aneurysms, improved after LV aneurysmectomy
}

\author{
Md Didar U1 Alam ${ }^{1}$, Muhammad Ihsan ${ }^{2}$ and Asma Syed ${ }^{1,2 *}$ \\ ${ }^{1}$ Brookdale Hospital Medical Center, 1 Brookdale Plaza, Brooklyn, NY 11212, USA \\ ${ }^{2}$ SUNY Downstate Medical Center, 450 Clarkson Ave, Brooklyn, NY 11203, USA
}

\begin{abstract}
Left ventricular aneurysm (LVA) is one of the common complications of myocardial infarction (MI) that can lead to death or serious morbidity [1]. We present a 71 YO woman PMH of CAD S/P LAD stent, HTN, DLD, RA myxoma, LV apical thrombus, s/p cardiac arrest and s/p single-chamber implantable cardioverterdefibrillator (ICD) on Mexiletine, who presented to hospital with multiple ICD shocks. Initial EKG showed a wide complex rhythm with a LBBB pattern. Patient was loaded with amiodarone and was continued on mexiletine. A magnet was placed temporarily over the ICD to avoid further shocks. Attempts with overdrive pacing during wide complex tachycardia were not successful. No significant electrolytes abnormalities or infections present during this admission. Both Echo on this admission and ECHO from last admission showed LV apical dyskinesis and aneurysmal deformity.
\end{abstract}

\section{Introduction}

Left ventricular aneurysm (LVA) is one of the common complications of myocardial infarction (MI) that can lead to death or serious morbidity [1]. The aneurysm may be asymptomatic or present as heart failure, sustained ventricular tachyarrhythmias, or arterial embolism. Radiofrequency catheter ablation (RFA) is commonly used in combination with an implantable cardioverter-defibrillator (ICD) and/or antiarrhythmic therapy for scar-related ventricular tachycardia (VT) associated with structural heart disease. In our case the patient had recurrent episodes of ventricular tachycardia for which she received multiple shocks from her ICD despite being on dual antiarrhythmic drugs. She was known to have an LV aneurysm, which was thought to be the likely source of her VT

\section{Case report}

A 71 YO woman with ischemic heart disease, s/p ICD for history of cardiac arrest on Mexiletine presented to ED after receiving multiple ICD shocks. On physical examination, she was tachycardic at 140 beats/min and BP was 139/75 mmHg. Initial EKG showed LBBB morphology. Laboratory data was significant for elevated troponin of 0.68 which was thought to be due to ICD shocks. While she was being evaluated she went into a wide complex tachycardia and was shocked by her ICD again. Patient was loaded with amiodarone and continued on mexiletine. ICD device interrogation revealed single lead ICD was functioning well and multiple episode of VT noted and appropriately detected and treated with ATP and shock therapy. On amiodarone, her arrhythmia burden appeared to have decreased. Patient had diagnostic Left heart catheterization(LHC) done, which demonstrated patent LAD stent and no significant coronary stenosis. Echocardiogram demonstrated LV apical dyskinesia and an aneurysmal deformity with LVEF mildly reduced (Figures 1 and 2).

Mexiletine was discontinued 2 days later. However, the subsequent day she started having episodes of monomorphic VT, rate around 160. Patient remained awake, alert at all times, no respiratory distress, stable hemodynamically, BP 130/72. She was continued on amiodarone and started on IV lidocaine. She was subsequently switched to oral amiodarone and mexiletine. The ventricular arrhythmia appeared to be well controlled on dual therapy and she was discharged home.

She was re-admitted to the hospital soon after with recurrent shocks. No significant electrolytes abnormalities were present. Decision was made to pursue radiofrequency ablation (RFA). She underwent EPS and successful ablation of LV apical on PO mexiletine $150 \mathrm{mg}$ BID for one month. Patient came back in follow up after 3 weeks with recurrent episodes of VT and shock. Decision was made to refer her for LV aneurysmectomy. She tolerated the procedure well without any complications. To date she has remained arrhythmia free without any anti-arrhythmics on board. (7 months later).

\section{Discussion}

The treatment of recurrent ventricular arrhythmia can be difficult especially in patients with ischemic cardiomyopathy and presence of LV aneurysms despite anti-arrhythmic medications [2]. The first line of treatment is anti-arrhythmic medications followed by radiofrequency ablation (RFA). RFA for incessant VT in the presence of a LVA is an effective treatment option [3]. However, in a case where the arrhythmia is not controlled despite these measures, surgery remains the treatment of choice. A true LVA is defined as a well delineated, thin, scarred, or fibrotic wall devoid of muscle or containing necrotic muscle and is a result of a healed transmural myocardial infarction (MI). The involved wall segment is either akinetic (without movement) or dyskinetic (with paradoxical ballooning) during systole. Aneurysms of the apex and anterior wall are more than four times as common as those of the

Correspondence to: Asma Syed, Brookdale Hospital Medical Center,1 Brookdale Plaza, Brooklyn, NY11212, USA, E-mail: asyed@bhmcny.org

Received: November 02, 2017; Accepted: November 23, 2017; Published: November 27, 2017 


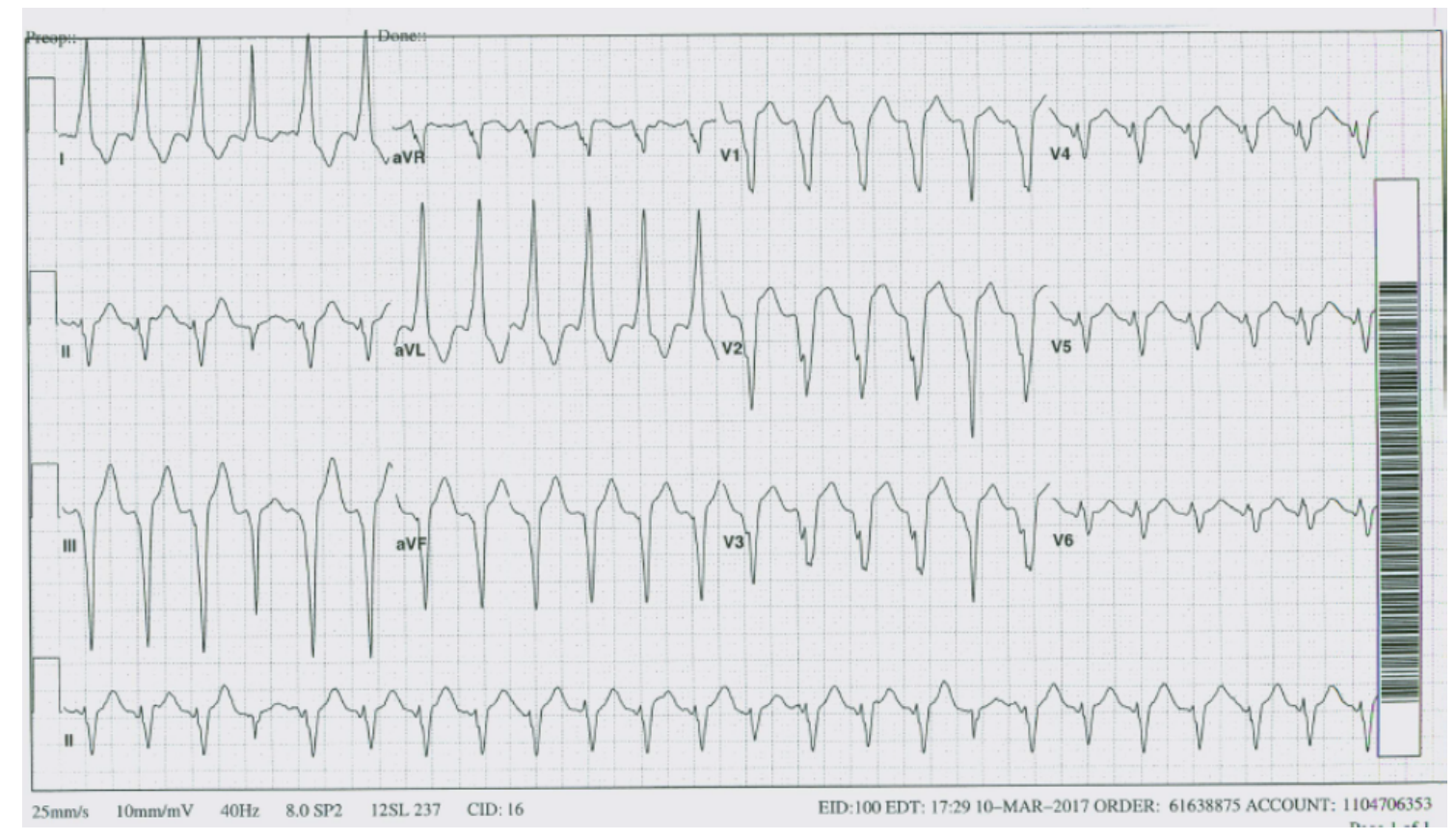

Figure 1. EKG Clinical Ventricular Tachycardia.

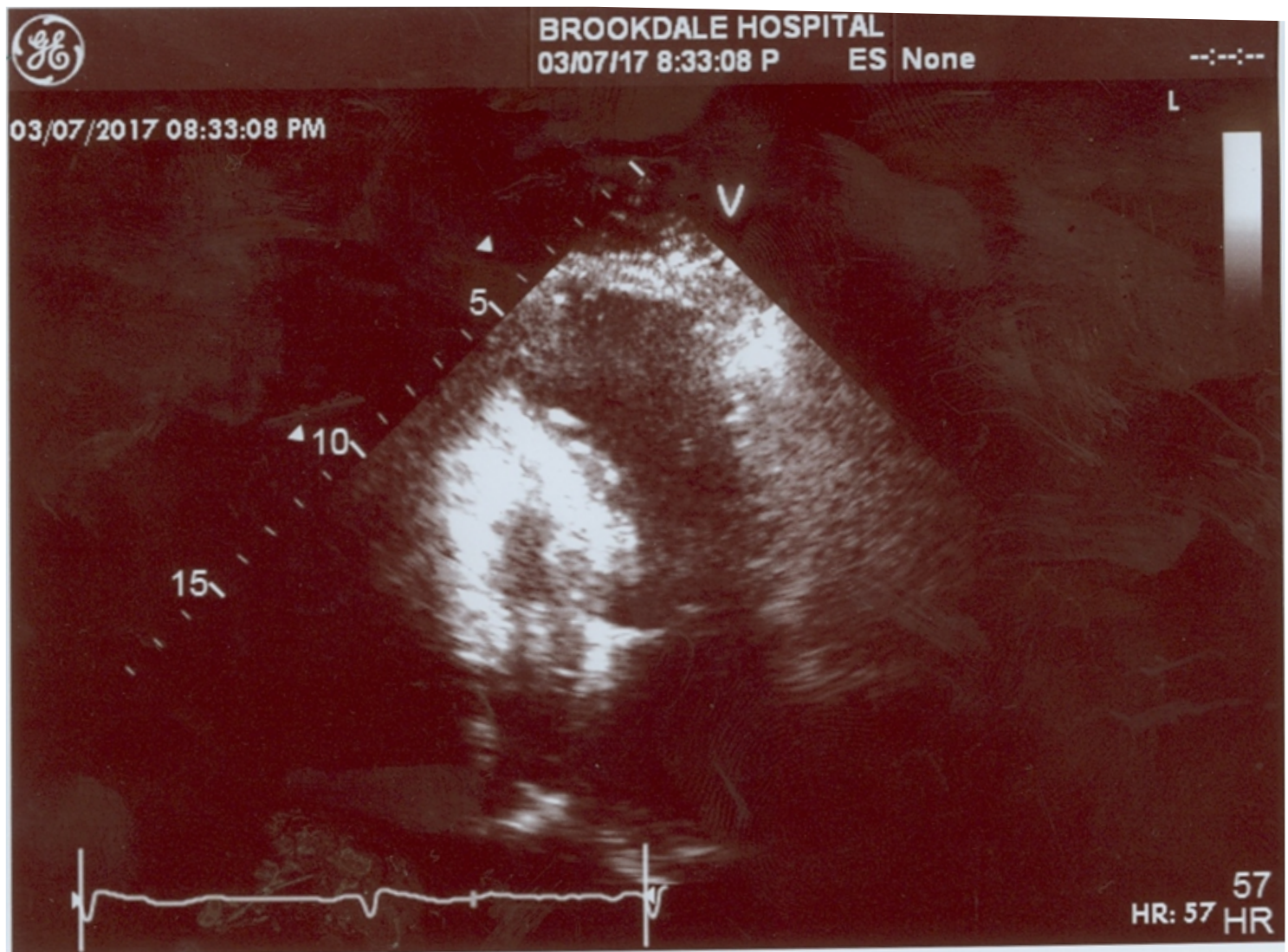

Figure 2. Echo showing left ventricular aneurysm 
inferior or anteroposterior walls. Ventricular arrhythmias, which can lead to sudden cardiac death, are common in patients with an LVA. Two principal mechanisms appear to contribute to ventricular arrhythmias: a). Myocardial ischemia and increased myocardial stretch can lead to enhanced automaticity or triggered activity. The myocardium located at the border zone is heterogeneous, consisting of a mix of fibrotic tissue, inflammatory cells, and damaged and disorganized muscle fibers. b). A reentrant tachycardia may develop around the border zone. We describe a case of recurrent VT in a patient with ischemic heart disease and LVA s/p ICD implant that failed to improve despite anti-arrhythmic therapy and RFA. RFA is commonly used in combination with an ICD and/or antiarrhythmic therapy for scar-related VT associated with structural heart disease. However, catheter ablation of VT in patients with LVA may not control the recurrent ventricular arrhythmia. In our case antiarrhythmic medications and RFA failed to control the arrhythmia and surgical resection of LV aneurysm provided the definitive treatment. [4-12]

\section{Conclusion}

This case report suggests that patients with LVAs post MI suffering from recurrent VT episodes not responding to maximally tolerated antiarrhythmic treatments and substrate modification, may benefit from LVAs resections. The number of patients referred for surgical resection is decreasing due to the advances in radiofrequency ablation and anti-arrhythmic medications. Aneurysmectomy has also become safer due to various advances and provides great benefit to patient with recurrent ventricular arrhythmias.

\section{References}

1. Mills NL, Everson CT, Hockmuth DR (1993) Technical advances in the treatment of left ventricular aneurysm. Ann Thorac Surg 55: 792-800. [Crossref]

2. Cain ME, Curtis AB1 (2016) Recurrent Ventricular Tachycardia--More Drugs or Bring Out the Catheter? N Engl J Med 375: 173-174. [Crossref]

3. Guo JR, Zheng LH, Wu LM, Ding LG, Yao Y (2017) Aneurysm-related ischemic ventricular tachycardia: safety and efficacy of catheter ablation. Medicine (Baltimore) 96: e6442. [Crossref]

4. Di Biase L, Burkhardt JD, Lakkireddy D, Carbucicchio C, Mohanty S, et al. (2015) Ablation of Stable VTs Versus Substrate Ablation in Ischemic Cardiomyopathy: The VISTA Randomized Multicenter Trial. J Am Coll Cardiol 66: 2872-2882. [Crossref]

5. Sacher F, Tedrow UB, Field ME, Raymond JM, Koplan BA, et al. (2008) Ventricular tachycardia ablation: evolution of patients and procedures over 8 years. Circ Arrhythm Electrophysiol 1: 153-161. [Crossref]

6. Kirklin JW, Barratt-Boyes B (1993) Cardiac Surgery. In: Left ventricular aneurysm, Kirklin JW, Barratt-Boyes B (Eds) Churchill Livingstone, New York. p.383.

7. Harken AH (1993) Surgical treatment of cardiac arrhythmias. Sci Am 269: 68-74. [Crossref]

8. Schlichter J, Hellerstein HK, Katz $\ln$ (1954) Aneurysm of the heart: a correlative study of one hundred and two proved cases. Medicine (Baltimore) 33: 43-86. [Crossref]

9. Waldo AL, Arciniegas JG, Klein H (1981) Surgical treatment of life-threatening ventricular arrhythmias: the role of intraoperative mapping and consideration of the presently available surgical techniques. Prog Cardiovasc Dis 23: 247-264.

10. Winkle RA, Alderman EL, Fitzgerald JW, Harrison DC (1976) Treatment of recurrent symptomatic ventricular tachycardia. Ann Intern Med 85: 1-7. [Crossref]

11. Tedrow U, Stevenson W G, and Benzaquen LR (2005) Apical left ventricular aneurysm presenting with malignant ventricular tachycardia responsive to aneurysmectomy. Heart 91: 623. [Crossref]

12. Pojar M, Harrer J, Omran N, Vobornik M (2014) Surgical cryoablation of drug resistant ventricular tachycardia and aneurysmectomy of postinfarction left ventricular aneurysm. Case Rep Med 207851. [Crossref]

Copyright: (C2017 Didar Ul Alam Md. This is an open-access article distributed under the terms of the Creative Commons Attribution License, which permits unrestricted use, distribution, and reproduction in any medium, provided the original author and source are credited. 